\title{
Addressing the Immunopathogenesis of Atopic Dermatitis: Advances in Topical and Systemic Treatment
}

\author{
Lawrence F. Eichenfield, MD,* and Linda F. Stein Gold, MD ${ }^{\dagger}$
}

\section{Abstract}

Several immunologic mediators-phosphodiesterase (PDE), interleukin (IL), small molecules, and Janus kinase-have been implicated in the pathogenesis of atopic dermatitis, and evidence has shown that blocking these mediators can help modify the disease process. Several new topical medications have been developed that target the enzyme PDE; crisaborole was recently approved by the US Food and Drug Administration (FDA) for the treatment of atopic dermatitis, and phase II studies have been completed on OPA-15406. The phase III clinical trial results of the systemic medication dupilumab, an inhibitor of the IL-4 receptor $\alpha$ subunit (which inhibits both IL-4 and IL-13 signaling), are currently being reviewed by the FDA. Semin Cutan Med Surg 36(supp2):S45-S48

(C) 2017 published by Frontline Medical Communications

\section{Keywords}

Atopic dermatitis; crisaborole; eczema; dupilumab; interleukin inhibition; OPA-15406; phosphodiesterase-4 inhibition

$\mathrm{T}$ he basic nature of atopic dermatitis (AD) - that it is the manifestation of an immune-mediated inflammatory process - has been recognized for several decades. Research has revealed that type $2 \mathrm{~T}$ helper cells $\left(\mathrm{T}_{\mathrm{H}} 2\right)$ play an important role in this process, as does dysfunction of the skin barrier. More recently, improved understanding of the pathogenesis of $\mathrm{AD}$ has prompted changes in our treatment strategies and spurred the development of new therapies. This article discusses two new topical medications that target the enzyme phosphodiesterase (PDE) (crisaborole and OPA-15406), current thinking on the role of systemic agents in $\mathrm{AD}$, as well

\footnotetext{
* Chief, Pediatric and Adolescent Dermatology, Professor of Dermatology and Pediatrics, Rady Children's Hospital, University of California,

San Diego School of Medicine, San Diego, California

Director of Dermatology Research, Henry Ford Health System, Detroit, Michigan

Publication of this CME/CE article was jointly provided by University of Louisville, Postgraduate Institute for Medicine, and Global Academy for Medical Education, LLC, and is supported by an educational grant from Anacor Pharmaceuticals, Inc. The authors have received an honorarium for their participation in this activity. They acknowledge the editorial assistance of Joanne Still, medical writer, and Global Academy for Medical Education in the development of this continuing medical education journal article.
}

Lawrence F. Eichenfield, MD, Advisory Board/Speaker: Valeant Pharmaceuticals North America LLC. Consultant: Anacor/Pfizer Inc., Eli Lilly and Company, Genentech, Inc., Otsuka America Pharmaceutical, Inc./Medimetriks Pharmaceuticals, Inc., Sanofi Genzyme/Regeneron Pharmaceuticals, TopMD, Valeant. Investigator: Sanofi Genzyme/Regeneron. Linda F. Stein Gold, MD, Consultant: Anacor. Grant/Research: Anacor, GlaxoSmithKline. Data Monitoring Committee: Otsuka.

Address reprint requests to: Lawrence F. Eichenfield, MD, Rady Children's Hospital, 8010 Frost Street, Suite 602, San Diego, CA 92123; leichenfield@ucsd.edu

1085-5629/13/\$-see front matter (C 2017 Frontline Medical Communications doi:10.12788/j.sder.2017.012 as new and emerging systemic agents. In recent years, several immunologic mediators-PDE, small molecules, and Janus kinase - have been shown to be important in the course of $\mathrm{AD}$, both because they have been implicated in the pathogenesis of $\mathrm{AD}$ and because of evidence that blocking these mediators can help change the disease process.

\section{PDE: A Novel Target for AD Treatment}

About 30 years ago, while studying leukocytes and macrophages in patients with AD and asthma, Hanifin and colleagues $^{1}$ discovered that activity of the enzyme PDE was increased and cyclic adenosine monophosphate (cAMP) activity was decreased in the presence of AD signs and symptoms. In normal cells, cAMP is present in a considerable quantity, and PDE can help mediate its consumption, but in inflammatory cells, PDE is overactive, cAMP is decreased, and a generalized overexpression of a number of proinflammatory cytokines ensues.

Many years after the work by Hanifin and colleagues was published, PDE was recognized as a potential target molecule for anti-inflammatory medications. Subsequent research determined that inhibition of PDE type 4 (PDE-4) results in increased levels of intracellular cAMP, the reduction of cytokine mediator release, and mitigation of the inflammatory processes involved in AD. PDE-4 inhibiting agents - several topicals and one systemic - have been investigated for the treatment of AD. The topical agent crisaborole was recently approved by the US Food and Drug Administration (FDA) for the treatment of AD. The other topical medication, OPA-15406 (also known as MM36), and the systemic PDE-4 inhibitor apremilast (currently approved for the treatment of moderate to severe plaque psoriasis and psoriatic arthritis) are being investigated as AD therapies that target the PDE-4 pathways.

\section{Crisaborole}

A new boron-based PDE-4 inhibitor, crisaborole, was approved by the FDA in December 2016 for the treatment of $\mathrm{AD}$. The incorporation of a boron ring into the cyclic structure of this agent allows effective penetration of the skin and access to target cells. In addition, the integration of boron into the molecule is thought to increase the stability of crisaborole and improve target binding and selectivity.

The safety and efficacy of crisaborole ointment $2 \%$ were evaluated in two identically designed randomized, doubleblind, vehicle-controlled phase III trials-referred to as AD-301 and AD-302 - involving a combined total of more than 1,500 patients with mild or moderate AD. ${ }^{2}$ The study populations $(n=759$ in $A D-301$ and $n=763$ in AD-302) included patients 2 years of age and older, with an average age of 12 years across both studies (range, 2-80 years). About one-third of the enrolled patients had mild AD and the rest had moderate disease, based on an Investigator's Static Global Assessment (ISGA) score. The mean affected body surface 
area (BSA) in AD-301 was $18.8 \%$ in the crisaborole group and $18.6 \%$ in the vehicle group; the mean BSA in AD-302 was $17.9 \%$ in the crisaborole group and $17.7 \%$ in the vehicle group.

The patients were assigned to receive crisaborole or vehicle in a ratio of 2:1. All subjects were instructed to apply the assigned study drug twice a day to all of the areas of AD identified at the baseline evaluation for 28 days.

The primary efficacy endpoint at day 29 was an ISGA score of clear or almost clear skin plus at least two grades of improvement over baseline. The investigators also evaluated time to success in the ISGA score, the percentage of patients who achieved a score of clear or almost clear, a decrease in the severity of AD signs, and the time to improvement in pruritus.

Significantly more patients in the active-treatment groups in both AD-301 and AD-302 achieved success on the ISGA score than did the patients in the vehicle groups: in AD-301, $32.8 \%$ vs $25.4 \%(P=0.038)$ and in $\mathrm{AD}-302,31.4 \%$ vs $18.0 \%$ $(P<0.001)$. Improvements in the secondary endpoints also were superior in the patients in the active-treatment groups (Figure 1 and Table 1).

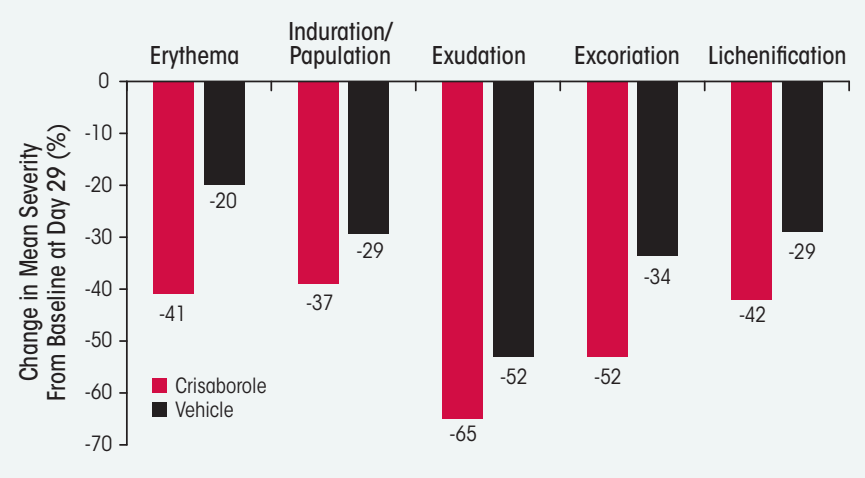

FIGURE 1 Crisaborole 2\% Ointment: Mean Change in Sign Severity in Phase III Studies.

Source: Paller AS, Tom WL, Lebwohl MG, et al. J Am Acad Dermatol. 2016;75:494-503.e4. ${ }^{2}$ Reprinted with permission.
Treatment-emergent adverse events were reported in about $11 \%$ of patients across both studies, and included AD and pain at the application site; a small percentage of patients had application site infection.

After completion of the 28-day phase III pivotal trials, patients had the opportunity to continue treatment by enrolling in a multicenter, open-label, long-term (48-week) extension safety study. ${ }^{3}$ A total of 517 patients opted to enroll. Patients were assessed for severity of their AD every 4 weeks and were treated with 4-week cycles of crisaborole as needed - ie, when severity was assessed as ISGA score of 2 or more (mild).

During this open-label extension and the pivotal studies, approximately $65 \%$ of patients had at least one treatmentemergent adverse event: severity of the events was mild or moderate, with most of these considered to be unrelated to treatment. Seven serious treatment-emergent adverse events were reported in the extension study, and none of these was considered to be treatment-related. ${ }^{3}$

Treatment-related adverse events included burning and stinging at the application site. No cases of application site atrophy, telangiectasia, or hypopigmentation were reported. ${ }^{3}$

\section{OPA-15406}

Phase II studies have been completed on another topical PDE-4 inhibitor, OPA-15406. In a randomized, double-blind, vehicle-controlled dose-finding study, Hanifin and colleagues ${ }^{4}$ compared two concentrations of OPA-15406 with vehicle in 121 patients between 10 and 70 years of age: OPA-15406 $0.3 \%(n=41)$, OPA-15406 1\% $(n=43)$, and vehicle $(n=37)$. The patients applied the study medication to the AD-affected sites twice daily for 8 weeks. The primary endpoint was an Investigator's Global Assessment (IGA) of 0 or 1, with a 2-grade reduction in AD severity at week 4.

This endpoint was achieved in the OPA-15406 1\% group $\left(P=0.0165\right.$ vs vehicle) (Figure 2A) ${ }^{4}$ In addition, the investigators noted that based on blood samples tested for OPA-15406, systemic absorption of this agent was negligible.

TABLE 1 Crisaborole 2\% Ointment: Summary of Phase III Studies Efficacy Results

\begin{tabular}{|c|c|c|}
\hline & $\begin{array}{c}\text { AD-301 } \\
\text { (crisaborole/vehicle) } \\
\mathrm{N}=503 / 256\end{array}$ & $\begin{array}{c}\text { AD-302 } \\
\text { (crisaborole/vehicle) } \\
N=513 / 250\end{array}$ \\
\hline \multicolumn{3}{|l|}{ Primary endpoint day 29} \\
\hline $\begin{array}{l}\% \text { ISGA success: Score of } 0 \text { (clear) or } 1 \text { (almost clear) } \\
\text { with } \geq 2 \text {-grade improvement }\end{array}$ & $\begin{array}{l}32.8 \% / 25.4 \% \\
\quad(P=0.038)\end{array}$ & $\begin{array}{l}31.4 \% / 18 \% \\
(P<0.001)\end{array}$ \\
\hline \multicolumn{3}{|l|}{ Secondary endpoints } \\
\hline ISGA of clear or almost clear (0 or 1) & $\begin{array}{l}51.7 \% / 40.6 \% \\
\quad(P=0.005)\end{array}$ & $\begin{array}{l}48.5 \% / 29.7 \% \\
(P<0.001)\end{array}$ \\
\hline \multicolumn{3}{|c|}{ Time to success in ISGA: Crisaborole achieved success earlier than vehicle-treated patients $(P<0.001)$. } \\
\hline $\begin{array}{l}\text { ISGA=Investigator's Static Global Assessment. } \\
\text { Source: Adapted from Paller AS, Tom WL, Lebwohl MG, et al. J }\end{array}$ & rmatol. 2016;75:494-503.e4.2 & \\
\hline
\end{tabular}




\section{Systemic Therapy}

The use of systemic treatments in children with AD whose disease is not adequately controlled with nonpharmacologic and topical treatments varies considerably among practitioners. This lack of treatment standardization is the result of insufficient evidence from clinical studies because pediatric patients are often excluded from enrollment. Two studies - one from the European Dermato-Epidemiology Network (EDEN) in Europe and the other from the Pediatric Dermatology Research Alliance (PeDRA) in the United States and Canada-surveyed clinicians to determine which of the currently available systemic medications they use in pediatric patients, and under what circumstances.

The European TREatment of Severe Atopic Eczema in Children Taskforce (TREAT) survey, conducted by EDEN, ${ }^{5}$ examined prescribing practices using currently available systemic agents. Among the 343 respondents $(89.2 \%$ of whom were dermatologists), $71 \%$ said they use systemic immunosuppression for children with severe AD. The first-line agents of choice were cyclosporine $(43.0 \%)$, oral corticosteroids $(30.7 \%)$, and azathioprine $(21.7 \%)$. The second-line agent of choice was cyclosporine (33.6\%), and the third-line choice was methotrexate (26.2\%).

In the United States and Canada, 133 members of the Society for Pediatric Dermatology responded to PeDRA's TREAT survey. ${ }^{6}$ In addition to determining prescribing practices, this survey sought to identify factors that discouraged the use of systemic agents. The survey showed that 113 respondents $(86.5 \%)$ said they use systemic therapy for severe AD in pediatric patients. The preferred first-line agents were cyclosporine $(45.2 \%)$, methotrexate $(29.6 \%)$, and mycophenolate mofetil (30.4\%). Methotrexate $(13.0 \%)$ and mycophenolate mofetil $(30.4 \%)$ were the preferred second-line agents, and azathioprine was the most commonly used third-line agent. Two factors were most commonly cited as issues that discouraged the use of systemic agents in pediatric patients: side effect profiles $(82.6 \%)$ and suspected risk for long-term toxicity $(81.7 \%)$.
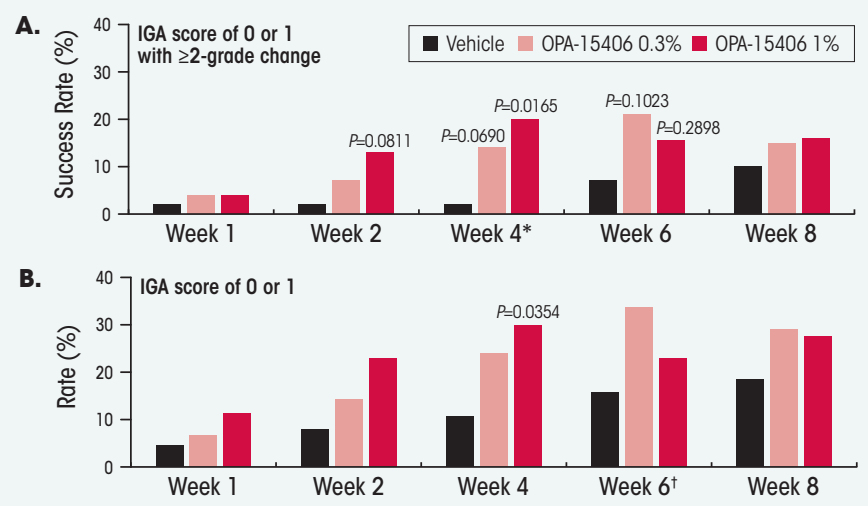

FIGURE 2 OPA-15406 Phase II Study Efficacy Data.

A. Mean success rates per visit defined as an Investigator's Global Assessment (IGA) score of 0 or 1 with 2-grade reduction from baseline. B. Mean rates of an IGA score of 0 or 1. $P$ values are for comparisons with vehicle.

*Treating for 8 weeks but primary endpoint at 4 weeks.

† Vehicle catches up after 6 weeks.

Source: Hanifin JM, Ellis CN, Frieden IJ, et al. J Am Acad Dermatol. 2016;75:297-305. ${ }^{4}$ Reprinted with permission.
At a crucial hearing of an FDA Advisory Committee on pediatric adverse events, eight professional societies, including PeDRA, the American Academy of Dermatology, and the Society for Pediatric Dermatology, endorsed a statement of advocacy regarding the need for studies of systemic agents in children with AD.

A number of new systemic agents currently are being investigated for the treatment of AD. Phase III studies on adult patients with AD have been completed on one of these agents, dupilumab, which is discussed below. Other systemic agents that target a variety of pathogenetic pathways (Table 2) are being evaluated in or have completed phase II clinical studies.

\section{Dupilumab}

A new systemic agent, dupilumab, is an inhibitor of the interleukin (IL)-4 receptor $\alpha$ subunit, which results in inhibition of both IL-4 and IL-13 signaling. Two identically designed phase III, 16-week trials of dupilumab monotherapy (SOLO 1 and SOLO 2) in adult patients with mild to moderate AD have been completed, and the results have been published. ${ }^{7}$

The studies involved 1,379 patients, 18 years of age and older, whose $\mathrm{AD}$ was not adequately controlled with topical agents or who were not candidates for topical medication. To be eligible for enrollment, patients were required to have a score of 3 or 4 on the IGA scale. Patients were also assessed at baseline using the Eczema Area and Severity Index (EASI) and other measures of $\mathrm{AD}$, although defined scores on these evaluations were not required for enrollment. Patients were randomized to three active-treatment arms or to a placebo group. The active-treatment groups received dupilumab, $300 \mathrm{mg}$ once weekly; dupilumab, $300 \mathrm{mg}$ every 2 weeks; or an initial loading dose of $600 \mathrm{mg}$ of dupilumab, followed by placebo for 16 weeks. The primary endpoint was the proportion of patients who had a score of 0 or 1 (clear or almost clear) in the IGA plus a reduction from baseline IGA of at least 2 points.

In the $300 \mathrm{mg} /$ week dupilumab groups in SOLO 1 and SOLO $2,37 \%$ and $36 \%$ of patients, respectively, achieved the endpoint IGA scores of clear or almost clear. In the groups

TABLE 2 Systemic Agents for Atopic Dermatitis in Ongoing or Completed Phase II Trials

\begin{tabular}{|c|c|}
\hline Drug Class & Agent \\
\hline PDE-4 inhibitor (oral) & Apremilast (oral) \\
\hline \multirow[t]{2}{*}{ IL-13 inhibitors } & Lebrikizumab \\
\hline & Tralokinumab \\
\hline IL-31 inhibitor & Nemolizumab \\
\hline \multirow[t]{2}{*}{ JAK inhibitors } & Tofacitinib \\
\hline & Baricitinib \\
\hline \multirow[t]{2}{*}{ Antileukotriene agents } & Q301 \\
\hline & QAW039 \\
\hline \multirow[t]{2}{*}{ Agents that target other pathways: } & H4 blockers \\
\hline & Liver X receptor antagonists \\
\hline $\begin{array}{l}\text { H=histamine; IL=interleukin; JAK=Janus ki } \\
\text { PDE-4=phosphodiesterase type } 4 \text {. }\end{array}$ & inase; \\
\hline
\end{tabular}



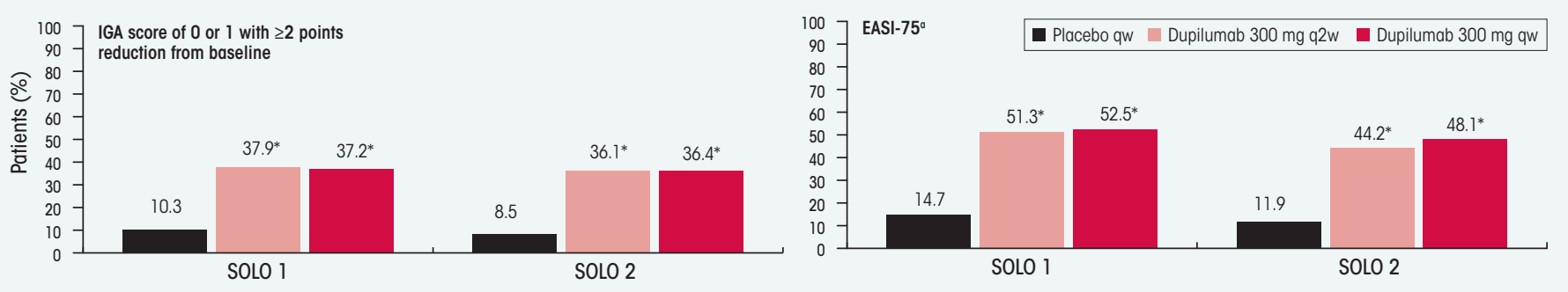

FIGURE 3 Dupilumab Efficacy in Moderate to Severe Atopic Dermatitis: Phase III Efficacy Results at 16 Weeks.

For binary outcomes, patients were categorized as nonresponders from the time rescue medication was used.

aCoprimary endpoint in European Union and Japan; key secondary endpoint in other regions.

${ }^{*} P<0.0001$ vs placebo.

EASI=Eczema Area and Severity Index; IGA=Investigator's Global Assessment.

Source: Adapted from Simpson EL, Bieber T, Guttman-Yassky E, et al. N Engl J Med. 2016;375:2335-2348. ${ }^{7}$

who received $300 \mathrm{mg}$ every 2 weeks, $38 \%$ and $36 \%$, respectively, achieved scores of clear or almost clear. By comparison, $10.3 \%$ and $8.5 \%$ of patients in the placebo groups in SOLO 1 and SOLO 2, respectively, had IGA scores of clear or almost clear. The differences in achievement of the primary endpoint were significantly different in all treatment-group vs placebogroup comparisons $(P<0.0001$ for all comparisons).

In the treatment groups who received $300 \mathrm{mg} /$ week dupilumab, improvements at week 16 over baseline in EASI scores were $72 \%$ and $69 \%$ in SOLO 1 and SOLO 2, respectively. In the patients who received $300 \mathrm{mg}$ dupilumab every 2 weeks, EASI improvements were $72 \%$ and $67 \%$, respectively, in SOLO 1 and SOLO 2. In the placebo groups, EASI improvements over baseline were $38 \%$ and $31 \%$ (the treatment-group vs placebo-group comparisons were statistically significant, at $P<0.0001$ for all comparisons).

Improvements of at least $75 \%$ in the EASI score (EASI 75 ) were seen in $52.5 \%$ and $48 \%$ of the groups who received $300 \mathrm{mg} /$ week dupilumab in SOLO 1 and SOLO 2, respectively. EASI 75 was achieved by $51 \%$ and $44 \%$ of patients who received $300 \mathrm{mg}$ dupilumab every 2 weeks in SOLO 1 and SOLO 2, respectively. In the placebo groups, $15 \%$ and $12 \%$ of patients achieved EASI 75 (comparisons with both treatment groups were statistically significant, $P<0.0001$ ). These 16 -week efficacy results are summarized in Figure 3. ${ }^{7}$

Adverse events occurred during the treatment period in $65 \%$ of patients in the dupilumab treatment groups in SOLO 1 and in $73 \%$ of patients in SOLO 2, vs $65 \%$ and $73 \%$ of patients in the corresponding placebo groups, respectively. Serious adverse events were seen in $1 \%$ and $3 \%$ of patients in the SOLO 1 and SOLO 2 dupilumab treatment groups, respectively, and in 5\% and 6\% in the corresponding placebo groups. The most commonly reported adverse events in the treatment groups were injection site reactions and conjunctivitis. No patients dropped out of the study because of an injection site reaction; one patient discontinued because of conjunctivitis.

A long-term (120 weeks), phase III, open-label extension study of dupilumab in pediatric patients with AD is currently recruiting subjects. The primary objective of the study is to assess the long-term safety of dupilumab in this population. The secondary objectives are to assess the long-term efficacy of the medication in pediatric patients with $\mathrm{AD}$, and to assess the trough concentrations of dupilumab in serum, as well as immunogenicity in this population after re-treatment with dupilumab. To be eligible, patients must be at least 6 years of age and younger than 18 years at the time of screening. In addition, patients must have participated in a previous phase II dupilumab study in patients with $\mathrm{AD}$ and satisfactorily completed the visits and assessments required in the previous study's protocol.

The primary endpoint is the incidence and rate of treatmentemergent adverse events from baseline to week 120 (the last study visit). Secondary outcome measures are the incidence and rate (events per patient-year) of serious adverse events and adverse events of special interest throughout the study period; the proportion of patients who achieve and maintain remission; the proportion of patients who achieve and maintain EASI 75 or better during the study; and the proportion of patients who achieve and maintain at least a $50 \%$ reduction in EASI (EASI 50) during the study.

\section{Conclusion}

Evolving understanding of the pathogenesis of AD, combined with the crucial clinical need for improved therapy, has created a fertile environment for drug discovery and development. New topical and systemic agents hold promise as important advances in AD management.

\section{References}

1. Hanifin JM, Chan SC, Cheng JB, et al. Type 4 phosphodiesterase inhibitors have clinical and in vitro anti-inflammatory effects in atopic dermatitis. $J$ Invest Dermatol. 1996;107:51-56.

2. Paller AS, Tom WL, Lebwohl MG, et al. Efficacy and safety of crisaborole ointment, a novel, nonsteroidal phosphodiesterase 4 (PDE4) inhibitor for the topical treatment of atopic dermatitis (AD) in children and adults. J Am Acad Dermatol. 2016;75:494-503.e4

3. Zane LT, Eichenfield LF, Call RS, et al. Long-term safety of crisaborole topical ointment, $2 \%$, in children and adults with mild-to-moderate atopic dermatitis. J Immunol. 2016;196(1 suppl):191.28. Abstract. http://www.jimmunol.org/ content/196/1_Supplement/191.28.abstract?sid=7d17786c-6ccc-4878-b44f252f6405158f. Accessed January 3, 2017.

4. Hanifin JM, Ellis CN, Frieden IJ, et al. OPA-15406, a novel, topical, nonsteroidal, selective phosphodiesterase-4 (PDE4) inhibitor, in the treatment of adult and adolescent patients with mild to moderate atopic dermatitis (AD): A phase-II randomized, double-blind, placebo-controlled study. J Am Acad Dermatol. 2016; 75:297-305.

5. Proudfoot LE, Powell AM, Ayis S, et al; European Dermato-Epidemiology Network (EDEN). The European TREatment of severe Atopic eczema in children Taskforce (TREAT) survey. Br J Dermatol. 2013;169:901-909.

6. Totri CR, Eichenfield LF, Logan K, et al. Prescribing practices for systemic agents in the treatment of severe pediatric atopic dermatitis in the US and Canada: The PeDRA TREAT survey. J Am Acad Dermatol. 2017;76:281-285.

7. Simpson EL, Bieber T, Guttman-Yassky E, et al; SOLO 1 and SOLO 2 Investigators Two phase 3 trials of dupilumab versus placebo in atopic dermatitis. $N$ Engl J Med. 2016;375:2335-2348. 
To get instant CME/CE credits online, go to http://tinyurl.com/meetingthechallengeofatopic. Upon successful completion of the online test and evaluation form, you will be directed to a Web page that will allow you to receive your certificate of credit via e-mail or you may print it at that time. If you have any questions or difficulties, please contact the Global Academy for Medical Education office at info@globalacademycme.com.

Questions: For each question or incomplete statement, choose the answer or completion that is correct. Circle the most appropriate response.

1. The characteristic, age-related anatomic distribution of atopic dermatitis in adolescents and adults includes the:
A. Antecubital fossa
B. Face (especially the cheeks and chin)
C. Neck
D. Popliteal fossa

2. Which of the following is a rare condition in the differential diagnosis of atopic dermatitis?
A. Acrodermatitis enteropathica
B. Contact dermatitis
C. Keratosis pilaris
D. Nummular dermatitis

3. In adults, the occurrence of should prompt consideration of other conditions in the differential diagnosis of atopic dermatitis.
A. Hand dermatitis
B. Late-onset signs and symptoms of atopic dermatitis
C. Pruritus
D. Wrist and ankle involvement

4. According to a study by Wen and colleagues, about $70 \%$ of patients with atopic dermatitis have a positive family history for
A. Atopic diseases
B. Gluten sensitivity
C. Peanut allergy
D. Seborrheic dermatitis in infancy

5. One strategy that may prevent atopic dermatitis in babies is regular use of
A. Antibacterial soaps
B. Bleach baths
C. Crisaborole
D. Emollients

6. One of the three main factors involved in the risk for persistence of atopic dermatitis into adulthood, identified recently by Kim and colleagues, is:
A. Disease in childhood that persists for up to 5 years
B. Greater compared to less severity of atopic dermatitis in childhood
C. Onset of disease earlier than 2 years of age
D. Resistance to topical calcineurin inhibitors

7. Evidence regarding bleach baths shows that they

A. Dry the skin when used more than twice weekly

B. Have both anti-inflammatory and anti-infective properties

C. Should be used according to standards set by the American Academy of Dermatology

D. Should not be used when skin is severely inflamed

8. The American Academy of Dermatology Guidelines for the treatment of atopic dermatitis recommend proactive use of topical as maintenance for relapse prevention, with intermittent periods of applications twice or three times weekly on areas that commonly flare.
A. Antimicrobial creams or ointments
B. Calcineurin inhibitors
C. Emollients
D. Interleukin inhibitors

9. Two new nonsteroidal topical agents, crisaborole (recently approved by the US Food and Drug Administration) and OPA-15406 (currently in clinical trials) for the treatment of atopic dermatitis target
A. Interleukin-13
B. Phosphodiesterase type 4
C. Tumor necrosis factor- $\alpha$
D. Type $2 \mathrm{~T}$ helper cells

10. is a novel systemic agent for the treatment of atopic dermatitis that targets the interleukin (IL)-4 receptor $\alpha$ subunit.
A. Dupilumab
B. Lebrikizumab
C. OPA-15406
D. Tofacitinib

The University of Louisville thanks you for your participation in this CME/CE activity.

All information provided improves the scope and purpose of our programs and your patients' care. 